\title{
Verbal pain descriptors used by patients with arthritis
}

\author{
SUSAN WAGSTAFF, ORIN V SMITH, AND PHILIP H N WOOD \\ From the ARC Epidemiology Research Unit, Stopford Building, University of Manchester, Oxford Road, \\ Manchester M13 9PT
}

SUMMARY Eighty patients with a diagnosis of either rheumatoid arthritis, localised osteoarthritis, or generalised osteoarthritis were asked to describe their pain by selecting words from a standardised list of pain descriptors. Words on this list were taken from the sensory class of pain descriptors found in the McGill pain questionnaire. Discriminant analysis was used to identify distinctive characteristics for each of these pain syndromes. On the basis of this analysis verbal responses for each patient were classified into one of the three diagnostic categories. Correct classification occurred in $65 \%$ of cases. An estimate of the technique's performance in prospective validation was derived by a split sample approach.

Key words: assignment, validation, diagnosis.

The meaning of pain has preoccupied humans through the centuries and is of special significance to those who treat the sick; medical practitioners use it as an aid to diagnosis. ${ }^{1}$ Pain is nevertheless still frequently considered in regard to a single attribute - variation in intensity. This concentration on one quality is reflected in the many measures of pain that have been developed. These may scale the experience numerically or record it graphically, ${ }^{2}$ but it is only intensity that is acknowledged rather than the qualitative dimensions.

Pain is difficult to describe; to communicate adequately what is perceived to another requires possession of a spontaneous vocabulary sufficient to translate feelings into words. One method of overcoming this difficulty is to invoke an external reference point, for example by describing what is experienced by analogy with sensations produced by known causes. Thus one may speak of a 'pricking' pain, selecting a word which relates to a common experience evoked by a pin.

A second way to facilitate pain description is to allow a patient to choose words from a standardised list of descriptors. This allows the person with limited spontaneous resources to draw on their larger passive vocabulary. A large number of such descriptors were collected by Melzack and Torgerson $^{3}$ to form part of the McGill pain ques-

Accepted for publication 9 November 1984.

Correspondence to Ms Susan Wagstaff, Department of Physiotherapy, Stepping Hill Hospital, Stockport, Cheshire. tionnaire (MPQ). A substantial part of the questionnaire relates to the three major classes of descriptor, $\bar{\partial}$ sensory, affective, and evaluative, that are used by patients to specify subjective pain experience. Each $\frac{}{\Phi}$ class is divided into subclasses which contain a number of words that are considered to be qualitatively similar. With the MPQ Dubuisson and Melzack $^{4}$ correctly assigned $77 \%$ of a large patient population into one of eight diagnostic categories, from the patients' pain language only. This suggests that there may be common word usage when describing pain of particular origins, and that there is a unique quality to the pain of many specific 8 diseases.

The primary aim of this study was to determine if $ᄋ$ similar usage of words could be detected in describ- $\rightarrow$ ing pain associated with more closely related diseases, namely, rheumatoid arthritis (RA), localised osteoarthritis (LOA), and generalised osteoarthritis (GOA), when compared with the conditions $N$ selected for inclusion in the Dubuisson study (men- N strual pain, arthritis, labour pain, degenerative disc 0 disease, toothache, metastatic carcinoma, phantom 0 limb pain, and postherpetic neuralgia. ${ }^{4} \mathrm{~A}$ secondary objective was to determine if there were specific $\stackrel{\mathscr{C}}{+}$ sensory qualities differentiating the pain experience $T$ of patients in our three diagnostic categories.

\section{Patients and methods}

Ninety-six patients attending rheumatology and orthopaedic outpatient clinics were interviewed, of $\varnothing$ 
whom 80 (27 male, 53 female) affirmed they were currently experiencing pain and were included in the study. Table 1 gives some characteristics of the participants. When asked for a description most of the patients experienced difficulty in finding words to characterise their pain adequately; only the word 'aching' occurred with any frequency. They were then offered a standardised list of words which described pain. This contained 17 words that had been selected from the MPQ, presented in a random arrangement (the word 'aching' was not on this list). A check was made to ensure that all the patients could see and read the printed words. Each was then asked to circle any word they considered adequately described their pain. No limit was put on the number of words that could be selected. After completing the selection the patients were asked if there were any other words which did not appear on our list but which they would like to add to describe their present pain.

Before the start of analysis the pain descriptors were allocated to appropriate subclasses designated by Melzack. ${ }^{5}$ This condensed the original 17 descriptors into seven subclasses, each containing words that are qualitatively similar. The subclasses were given the value zero if no word in that subclass was used, or a value equal to the scaling determined by Melzack and Torgerson ${ }^{3}$ as an indication of intensity. As all participants were allowed to select as many words as they deemed necessary, a method of analysis was required that would take account of this fact; simple cross tabulation methods were inappropriate. The statistical procedure selected was discriminant analysis. Thus an unknown individual could be correctly allocated to one of the three diagnostic groups based on their choice of words to describe pain. A technique was also required to identify words contributing most to the power of discrimination, thus revealing any redundancy in the data. Stepwise discriminant analysis has this capacity and also offers an estimate of the correct assignment rate if verbal responses had been used to predict diagnostic category.

\section{Results}

Table 2 shows the frequency with which words were selected. It is apparent that particular words were preferred by patients with the same diagnosis; throbbing and burning was the most popular choice

Table 1 Characteristics of participants in the study

\begin{tabular}{|c|c|c|c|c|c|}
\hline \multirow[t]{2}{*}{ Diagnostic group } & \multirow{2}{*}{$\begin{array}{l}\text { Number } \\
\text { of } \\
\text { subjects }\end{array}$} & \multicolumn{2}{|c|}{ Sex } & \multirow{2}{*}{$\begin{array}{l}\text { Median } \\
\text { age (yr) }\end{array}$} & \multirow{2}{*}{$\begin{array}{l}\text { Median } \\
\text { duration of } \\
\text { disease (yr) }\end{array}$} \\
\hline & & $M$ & $F$ & & \\
\hline Rheumatoid arthritis & 31 & 8 & 23 & 54 & 9 \\
\hline Localised osteoarthritis & 35 & 18 & 17 & 65 & 6 \\
\hline Generalised osteoarthritis & 14 & 1 & 13 & 63 & 5 \\
\hline
\end{tabular}

Table 2 Sensory subclasses of pain descriptors and frequency of word selection (expressed as a percentage with raw numbers in parentheses)

\begin{tabular}{|c|c|c|c|c|}
\hline Sensory subclass & Pain descriptor & $R A(31)$ & LOA (35) & $G O A$ (14) \\
\hline Temporal & Throbbing & $45(14)$ & 20 & $36(5)$ \\
\hline \multirow[t]{3}{*}{ Thermal } & Hot & 39 (12) & $6 \quad(2)$ & $36(5)$ \\
\hline & Burning & 42 (13) & $9 \quad$ (3) & $14(2)$ \\
\hline & Scalding & - & 3 (1) & - \\
\hline \multirow[t]{4}{*}{ Punctate pressure } & Stabbing & 13 (4) & 51 (18) & $29(4)$ \\
\hline & Boring & 13 (4) & 3 (1) & - \\
\hline & Pricking & 3 (1) & $6 \quad(2)$ & 21 (3) \\
\hline & Drilling* & - & - & - \\
\hline \multirow[t]{4}{*}{ Constrictive pressure } & Cramping & $23 \quad(8)$ & $29(10)$ & $29(4)$ \\
\hline & Gnawing & $26 \quad(8)$ & 9 (3) & $29(4)$ \\
\hline & Pressing & - & 6 (2) & 7 (1) \\
\hline & Pinching & 3 (1) & - & 7 (1) \\
\hline \multirow[t]{2}{*}{ Spatial } & Shooting & $19 \quad(6)$ & $26 \quad(9)$ & 7 (1) \\
\hline & Jumping & $10 \quad(3)$ & $6 \quad(2)$ & $14(2)$ \\
\hline \multirow[t]{2}{*}{ Incisive pressure } & Sharp & 23 (7) & $23 \quad(8)$ & $14(2)$ \\
\hline & Cutting* & - & - & - \\
\hline Brightness & Smarting & 7 (2) & $6 \quad(2)$ & 7 (1) \\
\hline
\end{tabular}

*These words were not selected by any participant. 
of words for patients with rheumatoid arthritis, and stabbing was most frequently chosen by those with localised osteoarthritis. Patients with generalised osteoarthritis made a more diverse selection of words. As the word aching was not included on the standardised list, it was excluded in subsequent analysis, but it was offered by 12 people, mainly those with localised OA. Two words, cutting and drilling, were not selected by any of the participants.

Fig. 1 illustrates the grouping produced by the discriminant functions, and indicates the efficiency of the procedure in correctly assigning an individual; distinct clusters would produce better discrimination than overlapping clusters. There is clustering of patients with RA and LOA, with some overlap, but no distinct grouping of the patients with GOA.

The results from a stepwise procedure indicated

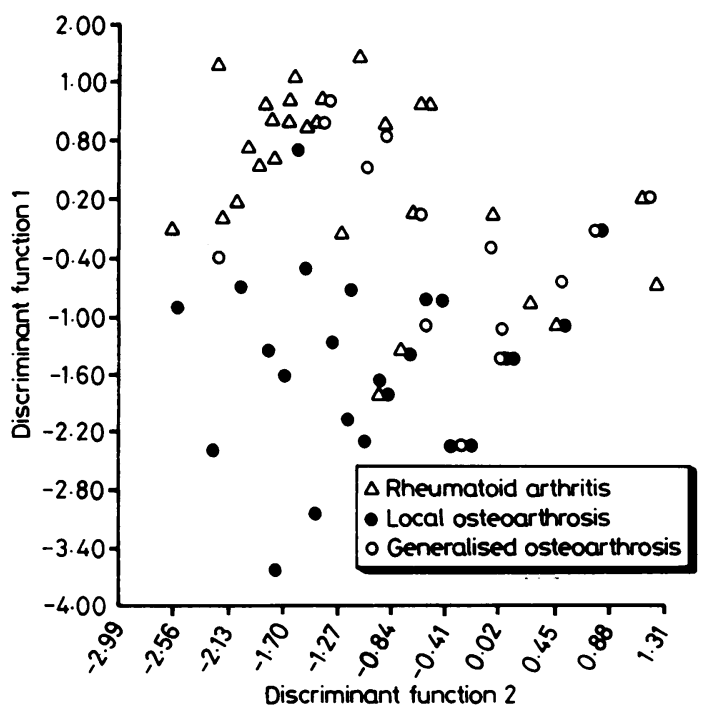

Figure Distribution of diagnostic categories in the space of the two discriminant functions. that two of the seven sensory subclasses of pain $\frac{}{\omega}$. descriptors, punctuate and thermal, accounted for most of the discriminating power (Table 3). $\stackrel{\vec{F}}{\vec{F}}$ Reanalysis with these two dominant subclasses? alone resulted in only a slight reduction in correct $\frac{}{\square}$ assignment. The addition of age and sex as discriminating variables contributed little to the correct $\mathbb{\otimes}$ assignment $(3 \cdot 8 \%)$. However, as expected, addition of the total number of joints involved as an extra ${ }^{\infty}$ discriminating factor improved correct assignment $\vec{O}$ from $56 \%$ to $60 \%$ (Table 3 ).

Patients with RA were most likely to select words $\stackrel{\vec{\omega}}{\circ}$ that implied a heat factor in their pain experience. Two new variables were therefore created, one? containing all the words suggesting heat (identified + as thermal in Table 2) and the other (non-thermal in Table 3) containing all other descriptors. Consi- ㅇ dered in isolation these two new variables producedo results which are an improvement on those based on? a fuller range of sensory subclasses.

It was appreciated that retrospective validation produced inflated estimates of correct assignment. To overcome this difficulty the original sample was $ळ$ randomly allocated into two groups. One of these ${ }^{\mathrm{c}}$ (group 1) was used to establish the discriminant rule, and in the second (group 2) the diagnosis was assumed to be unknown and was used to test the rule; correct assignment of patients in group 2 then⿳亠口冋口 gave an indication of the performance of the method $\stackrel{\mathbb{Q}}{\circ}$ on an independent sample. As the number of patients with GOA was small, it was decided not to 3 include them in this part of the analysis.

When all seven subclasses were considered there was very little difference between group 1 and group 2 in terms of correct assignment (Table 3). This was? also the case for the two dominant subclasses? thermal and punctuate alone and when the totalnumber of joints was added to those subclasses. Only for analyses involving thermal and nonthermal variables did a reduction occur in the correct assignment in group 2 relative to group 1 . I

Table 3 Classification of diagnosis from discriminant analysis

Subclass used in analysis
Correct classification (\%)

Using complete data set (retrospective)

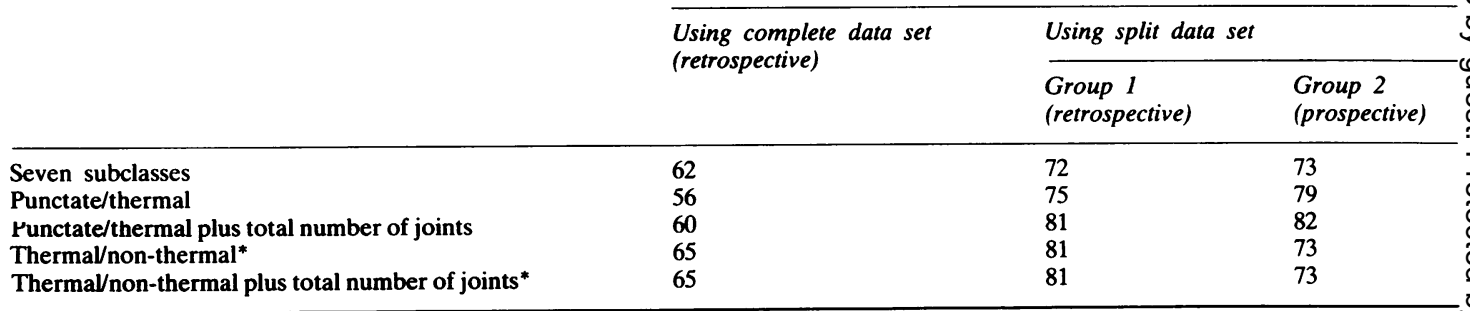

*Two new variables created by this study to reflect the heat factor in pain; these do not appear in the McGill pain questionnaire. 
The addition of the total number of joints caused noticeable improvement in the power of punctuate and thermal subclasses as predictors of diagnostic group, but this addition did not improve the power of thermal and non-thermal variables in diagnosis. This might suggest an association between the use of thermal words and polyarthritis and non-thermal words and monoarthritis. The improved performance of the technique in the split analysis is due to the exclusion of the GOA group.

\section{Discussion}

The sensation of pain is difficult to describe for, as Virginia Woolf ${ }^{6}$ says, '. . . let a sufferer try to describe a pain in his head to a doctor and language runs at once dry.' The word 'pain' is a linguistic lable, representing many different experiences.

Despite this, our results suggest that there are distinctive groups of words associated with specific pain syndromes, which in turn would indicate that there are unique qualities in the pain experienced by patients with RA and LOA. In LOA there tends to be a persistent ache, interspersed by intense episodes of stabbing pain provoked by movement of the affected joint, whereas in RA the pain is of a hot, burning nature. It was not possible to discern any distinctive quality of experience in patients with GOA, but their pain appears to be more diffuse. However, the number of subjects with this condition in our sample, 14, may have been insufficient to identify discriminating characteristics. When patients were unprompted, the word aching was offered by approximately a third of those with LOA, which would suggest that in subsequent studies this word should be included in any standardised list of pain descriptors.

Our results confirm the findings in the McGill study ${ }^{4}$ and, further, suggest that pain descriptors are sufficiently sensitive to discriminate between closely related pain syndromes. This could provide a useful tool for the physician. For example, if a patient with RA, who has persistently described their pain as being 'hot and throbbing', starts to use words more commonly associated with LOA, this may be an indication that secondary osteoarthritis is developing.

This method of inquiry therefore has a capacity to enhance understanding of the natural history of disease, especially when pain is a major feature. It may also be possible to use verbal pain descriptors as measures of outcome, with changes in word usage providing a measure of the effectiveness of a treatment regime. For instance, descriptors which indicate a high pain intensity may after treatment be replaced by words suggestive of a lesser intensity.

\section{References}

1 Agnew, D C, Merskey H. Words of chronic pain. Pain 1976; 2: 73-81.

2 Scott J, Huskissson E C. Graphic representation of pain. Pain 1976: 2: $175-84$.

3 Melzack R. Torgerson W S. On the language of pain. Anesthesiology 1971: 34: 50-9.

4 Dubuisson D. Melzack R. Classification of clinical pain descriptors by multiple group discriminant analysis. Exp Neurol 1976: 51: 480-7.

5 Melzack R. The McGill pain questionnaire. Pain 1975; 1: 277-99.

6 Woolf V. On being ill. In: Melzack R, ed. The puzzle of pain. London: Penguin. 1977: 45. 